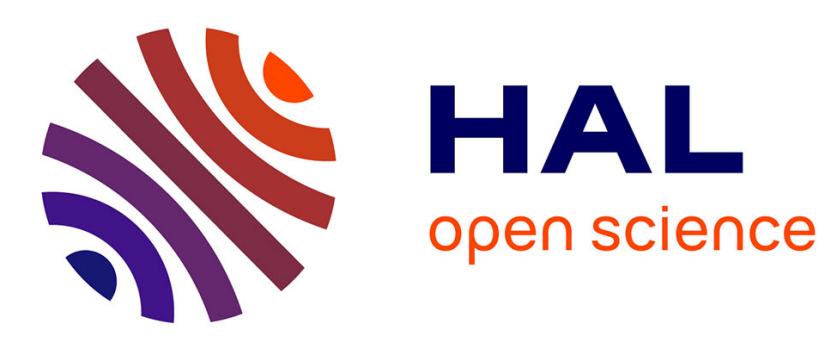

\title{
Arc root interaction with the electrode: a comparative study of 1D-2D axi symmetric simulations
}

\author{
M'Hammed Abbaoui, A. Lefort
}

\section{To cite this version:}

M'Hammed Abbaoui, A. Lefort. Arc root interaction with the electrode: a comparative study of 1D-2D axi symmetric simulations. European Physical Journal: Applied Physics, 2009, 48 (1), pp.1-9. 10.1051/epjap/2009134. hal-00505544

\section{HAL Id: hal-00505544 https://hal.science/hal-00505544}

Submitted on 24 Jul 2010

HAL is a multi-disciplinary open access archive for the deposit and dissemination of scientific research documents, whether they are published or not. The documents may come from teaching and research institutions in France or abroad, or from public or private research centers.
L'archive ouverte pluridisciplinaire HAL, est destinée au dépôt et à la diffusion de documents scientifiques de niveau recherche, publiés ou non, émanant des établissements d'enseignement et de recherche français ou étrangers, des laboratoires publics ou privés. 


\title{
Arc Root Interaction with the Electrode: a Comparative Study of 1D-2D Axi Symmetric Simulations
}

\author{
M. ABBAOUI and A. LEFORT
}

LAEPT - Clermont University-CNRS, Blaise Pascal University, Physique 5, 24 avenue des Landais, F63177 AUBIERE Cedex

Received: date / Revised version: date

\begin{abstract}
Two simulation methods of the energy transmitted by the arc roots to the electrode material are described and their results are compared together and with these found by other authors. About a copper electrode the time phase evolutions are given when a constant energy flux is applied to the contact surface. The obtained results are better for vacuum and small current. The cathode and anode result discussions lead to propositions to improve arc root models.
\end{abstract}

PACS. PACS-key 52.40.Hf - PACS-key Plasma-material interactions; boundary layers effects

\section{Introduction}

The existence of electric arc discharge needs transition zones between the thermal plasma and the metallic electrodes, the emission of electrons (cathode) and their capture (anode) is not possible without energy exchange and its consequences: the electrode surface transformations. It is necessary to understand the material transformations and to use them in the best conditions; the goal is not the same in welding or in breaker contacts. At the cathode arc root, the emitting centres are disconnected in arc vacuum and adjacent in others cases, they are called fragments, the present study is relative to one fragment cathode. The an- ode arc root exists only to collect column electrons that transmit their energy to the electrode, as in the fragments they have a circular shape. To study material evolution two simulation methods are used:

- the 1D simulation represents a first approach and gives the first results necessary to justify the validity of our work,

- the 2D axi symmetric simulation is firstly technically verified by comparison with $1 \mathrm{D}$ results.

The choice of a two presentation methods is to show the importance (or not) of the improvement given by the second method, the 1D simulation needs calculation times 
lower than the $2 \mathrm{D}$ one, there is a factor three for the time calculation that depends of the time and space steps.

\section{Simulation method}

The arc root, with the hypothesis of a circular shape, sends to the metal electrode supporting it a supposed constant surface energy flux. To obtain the material phase and temperature evolutions in each point, we solve the enthalpy form heat equation:

$$
\frac{\partial H}{\partial T}=\operatorname{div}(k \vec{\nabla} T)+\text { Source }
$$

$\mathrm{H}, \mathrm{k}$ and $\mathrm{T}$ represent respectively the material enthalpy, the thermal conductivity and the temperature. The term "Source" is taken equal to zero. The constant energy flux $\mathrm{W}$ is applied at the time $t=0$ and maintained till $t=\tau$. Energy is provided to the domain $\Omega_{t}$ through the surface $\Gamma_{W_{t}}$, equation (1) is transformed into an equation of Stefan:

$$
\begin{array}{r}
\int_{\Omega_{t}} \frac{\partial H(r, t)}{\partial t} \phi(r) d r+\int_{\Omega_{t}} \nabla \beta(H(r, t)) \nabla \phi(r) d r= \\
\int_{\Gamma_{W_{t}}} W(r, t) \phi(r) d s
\end{array}
$$

with:

$$
\beta(H)=\int_{T_{0}}^{T} k(u) d u
$$

$T$ and $T_{0}$ are respectively the temperature at the moment considered and at the time $t=0, \phi$ is a regular function with real values, necessary to discretize the problem,

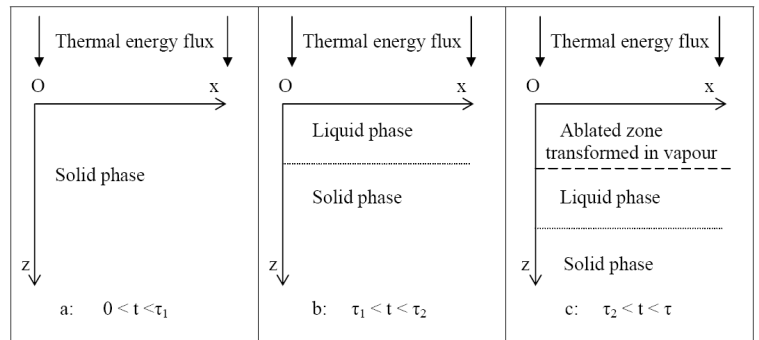

Fig. 1. 1D simulation: the thermal energy flux is applied to the infinite plan $\mathrm{Ox}, \mathrm{Oy}$, the energy dissipates only in z-axe direction. In the $\mathrm{b}$ and $\mathrm{c}$ case the thermal flux is applied on a liquid phase. At $t=\tau_{1}$ and at $t=\tau_{2}$ respectively the liquid and vapour phase appear.

$d s$ is an element of the surface $\Gamma_{W_{t}}, \vec{r} \in \Omega_{t}$ and $s \in \Gamma_{W_{t}}$.

A finite element method is used with moving boundaries and ablation to simulate the time evolution of the solid liquid limit and of the liquid vapour limit. When a node enthalpy is higher than the vaporisation enthalpy this node is removed and the energy flux is transmitted to the next liquid (or solid) node.

\subsection{D simulation}

The figure 1 summarizes a method detailed in the paper of Rossignol [1]. The surface energy flux $W$ applied on the plan surface Ox, Oy is transmitted to the material only in the $\mathrm{z}$-axe direction, the time duration is equal to $t_{\max }=$ $\tau$, and we are interested only by the material evolution during this time $t_{\max }$.

The time interval $\left[0, t_{\max }\right]$ is divided on a regular basis with the time step $t_{p}=\frac{t_{\max }}{M}$ considered and moments are 
defined by $m . t_{p}$, with $m=0 \ldots M$. Interval space $\left[0, z_{\max }\right]$ is divided into $N$ intervals on each of which is expressed the approached function:

$$
H_{h}^{m}=\sum_{j=0}^{N} H_{j}^{m} \phi_{j}
$$

Assuming known $H_{h}^{m}$ functions, equation (2) leads to the values of $H_{h}^{m+1}$ :

$$
\begin{array}{r}
\int_{\left[z_{m}, z_{\text {max }}\right]} \frac{H_{h}^{m+1}-H_{h}^{m}}{\tau} \phi(z) d z+ \\
\int_{\left[z_{m}, z_{\text {max }}\right]} a\left(H_{h}^{m}\right) \frac{d H_{h}^{m+1}}{d z} \frac{d \phi(z)}{d z} d z= \\
\int_{\left[z_{m}, z_{\max }\right]} W \cdot \phi\left(z_{m}\right) \cdot \phi(z) d z
\end{array}
$$

$a\left(H_{h}^{m}\right)$ represents the magnitude of the derivative of the function $\beta\left(H_{h}^{m}\right)$. The element $z=0$ is removed from the computational domain when the enthalpy $H_{h}^{m+1}$, which corresponds to it becomes greater than the enthalpy $H_{v a p}$ needed for the vaporization of this element. At a moment $m . t p$ several elements have been removed by ablation, the initial area $\left[0, z_{\max }\right]$ is reduced to $\left[z_{m}, z_{\max }\right]$. At $t=0$ the specific enthalpy is supposed to be equal to $H_{0}$ at each point of the considered volume, in the program we considered only the points of the Oz-axe, the points with coordinate $(x, y, z)$ present the same specific enthalpy or temperature value when $z$ is fixed, the space and time evolutions are only in the $z$ coordinate. The studied domain is limited by the coordinates $z_{0}=0$, and $z=z_{\max }$, the second limit determines the calculated length $l=z_{\max }-$ $z_{0}$, and it obeys to the condition $H=H_{0}$ during the energy flux duration $t_{\max }$. The length domain $l$ is divided in 3000 steps with different values, the first 2000 steps begin-

Fig. 2. 2D axi symmetric simulation: the thermal energy flux is applied to a radius $r_{a r}$ circle surface on the plane surface of an axe $\mathrm{Oz}$ and radius $r_{\max }$ cylinder. The energy dissipates in r-axe and z-axe directions. At $t=\tau_{1}$ and at $t=\tau_{2}$ respectively the liquid and vapour phase appear.

ning at $z=0$ are shorter and equal to $h_{1}$, than the 1000 steps finishing at $z=z_{\max }$ and equal to $h_{2}$. The step time discretization $t_{n}$ is chosen constant and equal to $\frac{\tau}{N}$ with $N=10^{6}$ to avoid computation difficulties.

To compare the obtained results with experiment or with the $2 \mathrm{D}$ axi symmetric simulation results, we used the coordinate $\mathrm{r}$ in the plan $O x, O y$. In this case the considered volumes are cylinders with the axis of symmetry $O z$.

\subsection{D axi symmetric simulation}

To solve equation (1) the numerical method of finite element described in 2.1 is used, but in this case presented in figure 2 the material is a finite cylinder. The $\mathrm{z}$-axe represents the symmetric axe of the cylinder and the r-axe situates the points along a radius perpendicular to the axe z-axe. 
The arc root is supposed motionless, this hypothesis is satisfied by the commonly admitted reasons:

- at the cathode the life time of the fragment is very short (less than 100ns),

- at the anode the spot moves slowly, or presents a short life time if it moves quickly (in the presence of a magnetic field).

Joule heating is neglected : a simple calculation shows that the produced energy in the electrode contracted zone of the current lines is very small in comparison with the transmitted thermal energy. About the enthalpy function versus temperature, the same hypothesis like in $1 \mathrm{D}$ simulation is made: the phase changes (solid-liquid and liquidvapour) are made to be continuously on a small gap of temperature $\left(10^{\circ} \mathrm{C}\right.$ for example).

The surface energy flux $\mathrm{W}(\mathrm{r})$ is applied at $t=0$ on a circular surface with centre $\mathrm{O}$ and radius $r_{a r}$ during a time $t_{\max }=\tau, \mathrm{W}(\mathrm{r})$ can be variable with the radius $\mathrm{r}$ and with the time $t$; in the results presented in the next paragraphs $\mathrm{W}(\mathrm{r})$ is constant during the time $\tau$. At the time $t=0$ the specific enthalpy for each node of the cylinder is equal to $H_{0}$, then, for $t>0$ the energy is transmitted in the z-axe and r-axe directions providing a change in the enthalpy values in each point of the considered volume. Starting from equation (2) and performing the same operations of discretization for an $1 \mathrm{D}$ representation, we get:

$$
\begin{array}{r}
\int_{\Omega_{r, z}} \frac{H_{h}^{m+1}-H_{h}^{m}}{\tau} \cdot \phi \cdot r . d r . d z \\
+\int_{\Omega_{r, z}} a\left(H_{h}^{m}\right) \nabla d H_{h}^{m} \cdot \nabla d \phi \cdot r \cdot d r . d z
\end{array}
$$

$$
=\int_{\Omega_{r, z}} W \cdot \phi_{\Omega_{r, z}} \cdot \phi \cdot r \cdot d s
$$

with $\phi=\phi(r, z)$. The initial field consists of triangles $\mathrm{K}$ (Fig. 3), but because of the axisymmetry, a field $\mathrm{K}$ will be deleted when the following condition will be satisfied:

$$
\int_{K} H_{h}^{m+1} \cdot r \cdot d r \cdot d z>\int_{K} H_{v a p} \cdot r \cdot d r . d z
$$

and the initial field is reduced to the area noted $\Omega_{r, z}$. The solution of equation (6) is obtained by solving a linear system.

At the instant $t=\tau_{1}$ the specific enthalpy reaches a value equal to the specific liquefaction enthalpy and the first liquid volume appears, in this case the figure 2-b shows that the surface energy flux is applied to the liquid and to the solid and one can obtain the time evolution of the depth, radius and volume of the liquid phase. At the instant $t=\tau_{2}$ the same phenomena is obtained with the vaporization enthalpy, the corresponding meshes and nodes are removed and the first vaporized volume appears, and one can obtain the time characteristic evolution of this volume.

The figure 3 shows the shape of the meshes used; in the results presented in paragraphs 3 and 4 , the cylinder radius $r_{\max }$ and height $z_{\max }$ are divided in 125 steps giving 16400 nodes. The time step is chosen equal to $t_{\max } / 1000$ to obtain correct results with a reasonable PC time computation.

In the $1 \mathrm{D}$ simulation case, the energy propagates only in the z-axe direction, the obtain different zones are cylinders (figure 4a), their volume evolution gives results about 


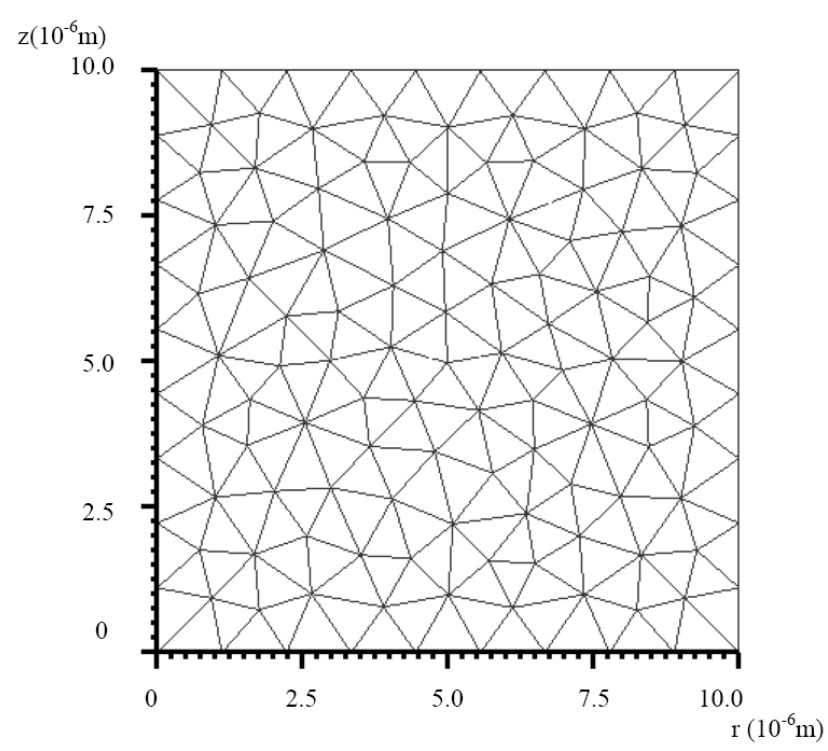

Fig. 3. An example of the meshes used in the $2 \mathrm{D}$ axi symmetric simulation.

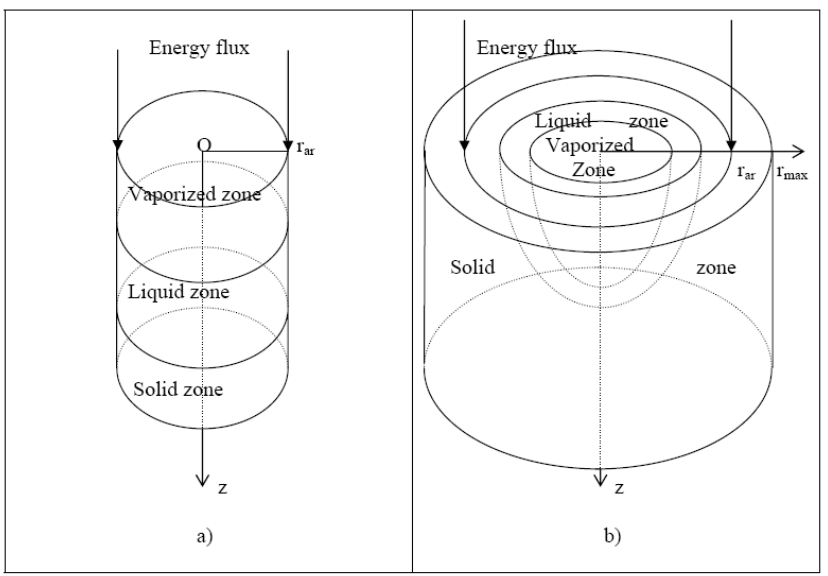

Fig. 4. Geometry of the contact with the two phases (solid and liquid), and the ablated zone by vaporization: a) 1D case: the zones are cylinders ; b) 2D axi symmetric case.

contact erosion by vaporisation and about the liquid volume obtained. In the $2 \mathrm{D}$ axi symmetric simulation the same results appear (figure $4 \mathrm{~b}$ ), the shapes of the obtained volumes are different, this fact is due to the energy propagation in z-axe and in radial-axe directions.

\section{Cathode arc root}

At the cathode surface the classical [2] power flux balance gives:

$$
P_{i}=P_{H}+P_{r}+P_{e}
$$

$P_{i}$ is the surface power flux delivered to the cathode surface by the ions coming from the plasma sheath, $P_{i}$ is directly proportional to the cathode current density $J_{c}$. The power flux $P_{e}$ corresponds to the cooling effect produced by electron emission, generally it takes low values [3] and one can neglect it comparatively to the values taken by $P_{i}$. The surface power flux $P_{r}$ dissipated by the cathode surface radiation takes also low comparative values; it is the result of cathode surface temperature which is equal to the metal vaporization temperature. Under these considerations equation 8 simplifies, and the power flux $P_{H}$ transmitted to the cathode material is equal to the incoming power flux $P_{i}$. Using the values given by Rakhovskii [4] about the proportionality coefficient necessary to obtain $P_{i}$ and making with Jüttner [5-6] the hypothesis of a cathode fragment of $5 \mu m$ radius and transporting a 10A current, the values obtained about $P_{i}$ gives $W=5 \times 10^{11} \mathrm{Wm}^{-2}$. The cathode presented results about copper are obtain with these values of $W$ and $r$, the time duration $t_{\max }$ is taken equal to $100 \mathrm{~ns}$, it corresponds to the maximum cathode fragment life. 


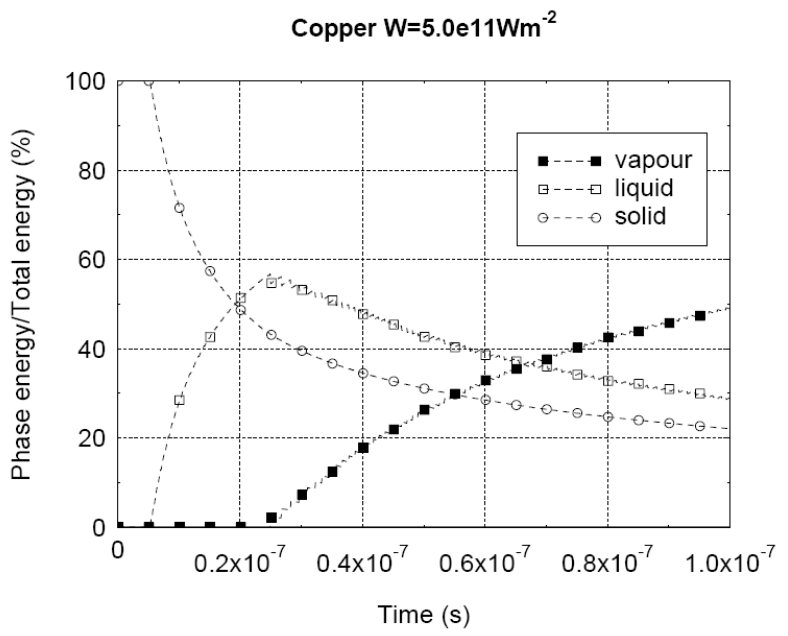

Fig. 5. The three phase energy partition versus time in the contact (1D case).

\subsection{D simulation results}

The figure 5 gives the evolution with time of the energy partition. At the beginning the whole energy is used to heat the material by conduction till $t_{c l i q 1 D}=5.4 n s$, at this time the liquid phase appears. The energy is now consumed by the two phases, the solid part decreases and the liquid part increases and reaches a maximum value at $t_{\text {cvap } 1 D}=25 n s$, at this time the vapour phase appears. The energy is now dissipated by vapour production, by solid liquid transformation and by thermal conduction in the liquid and solid phases. The part of energy used to the production of the vapour grows, it takes a value near $50 \%$ at the end of the spot life.

On the figure 6 the temperature repartition in the material is represented at different times. The vaporized and liquid parts appear clearly, the temperature repartition in the liquid is linear, it is the result of the imposed and constant temperatures $T_{v a p}$ and $T_{l i q}$ at each end. The tem-

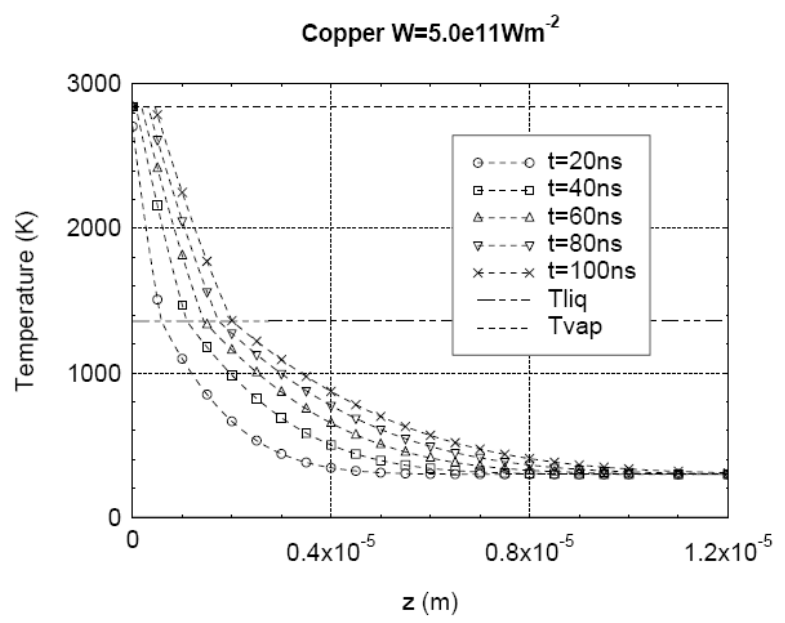

Fig. 6. Contact cathode temperature repartition along $\mathrm{z}$ axe at different instants (1D case).

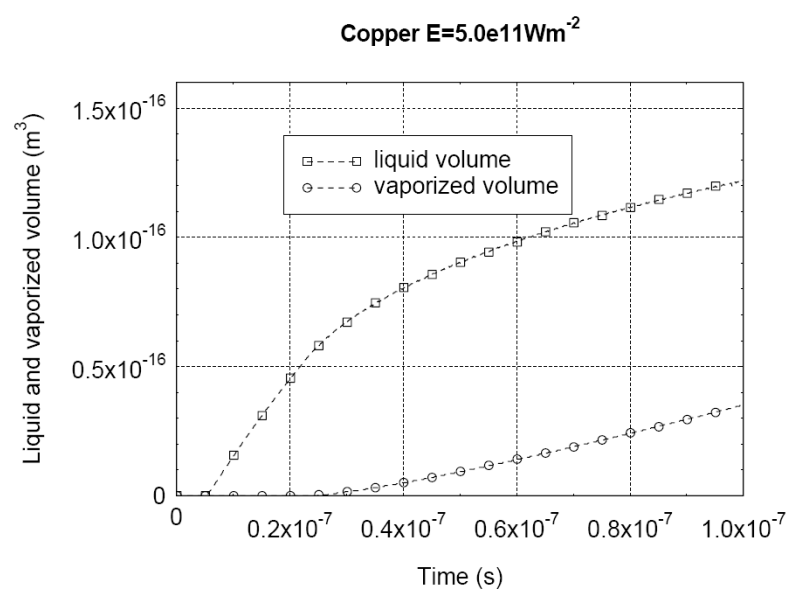

Fig. 7. Time evolution of the liquid and vaporized volume at the cathode contact (1D case).

perature distribution in the solid part is well known and classical. At $t=100 \mathrm{~ns}$ the depth of the vaporised material is $z_{\text {cvap } 1 D}=0.4 \mu \mathrm{m}$ and the thickness of the liquid phase takes the value $z_{\text {cliq } 1 D}-z_{\text {cvap } 1 D}=1.6 \mu \mathrm{m}$.

The simulation gives the time evolution of the vaporized and liquid zones along $\mathrm{z}$ axis, knowing the fragment radius $r_{\max }=5 \mu \mathrm{m}$, it is then possible to obtain the liquid and vaporized volumes (figure 7 ). The curve about the va- 


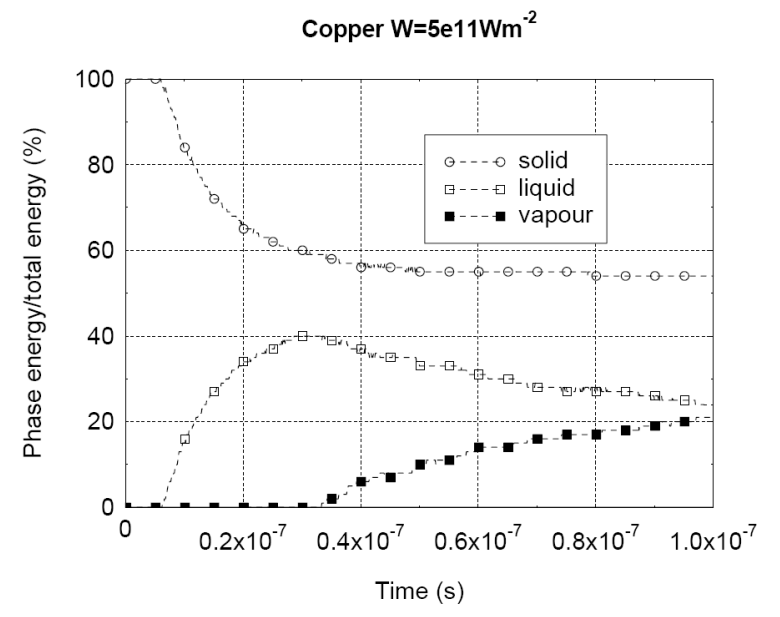

Fig. 8. The three phase energy partition versus time in the cathode contact (2D axi symmetric case).

porized volume is practically linear versus time, the slope multiplied by copper density and divided by the electric charge travelled through the fragment gives the vapour erosion rate $E_{r c 1 D}=415 \mu \mathrm{g} / \mathrm{C}$.

\subsection{D axi symetric simulation results}

At $t_{c l i q 2 D}=6.1 n s$ (figure 8 ) the liquid phase appears and the part of energy absorbed by this liquid phase grows till a maximum value, about $40 \%$ at $t_{c v a p 2 D}=32 n s$, corresponding to the vaporization beginning. One can see on figure 8 that with the vapour apparition the solid energy part decreases very slowly to the value $54 \%$, in this time interval the parts used to the vaporization and to the liquid phase vary in opposite direction. At the end of the fragment life the energy part in the vapour is $20 \%$.

The figure 9 gives the temperature variations along zaxe at different times, the evolution is similar with $1 \mathrm{D}$ results (figure 6 ). After $t=40 n s$ (figure 10) the radius of the vaporized zone presents a very slow evolution between

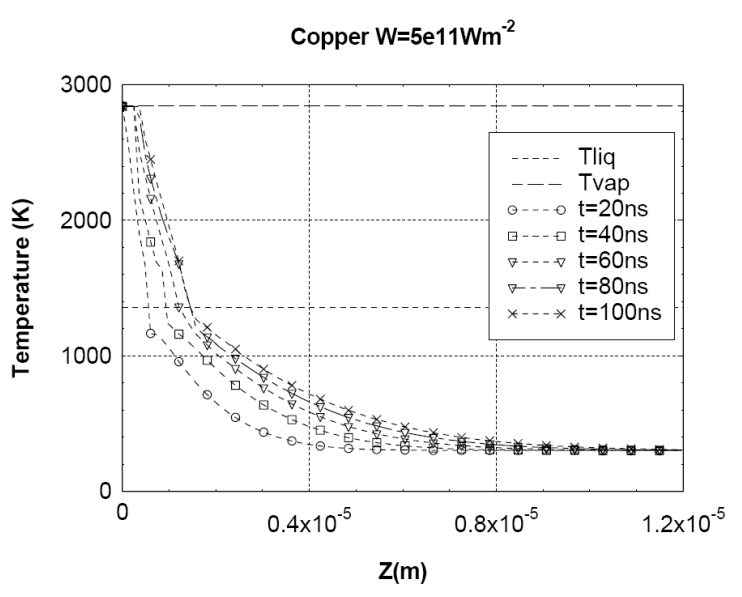

Fig. 9. Cathode temperature repartition along z-axe at different instants (2D axi symmetric case).

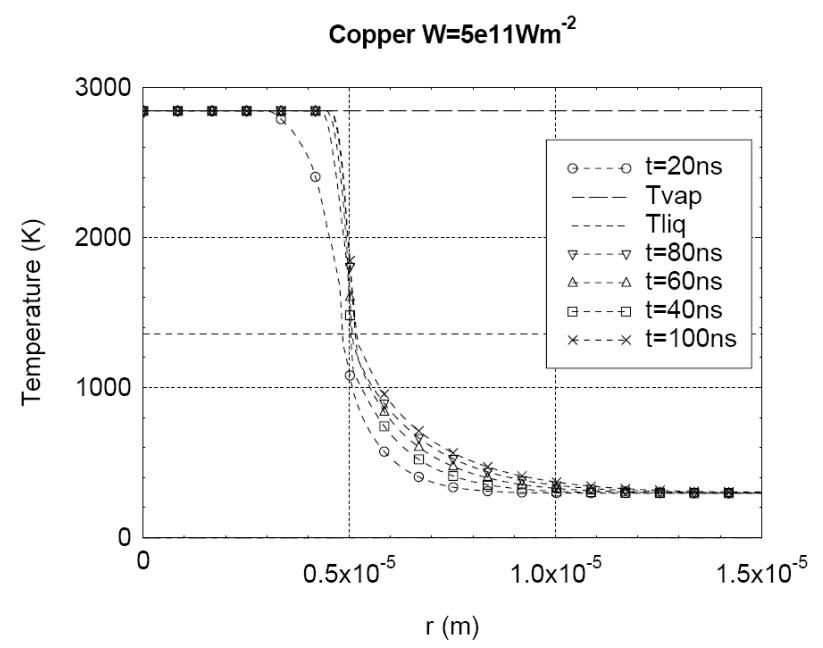

Fig. 10. Temperature repartition along r-axe, on cathode contact surface at different instants (2D axi symmetric case).

$r_{40 n s}=4.3 \mu m$ and $r_{100 n s}=4.6 \mu m$, the liquid phase growth is in major part directed in the z-axe direction. The length of the liquid phase in the r-axe direction on the electrode surface at $t=100 \mathrm{~ns}$ is $r_{c l i q 2 D}-r_{\text {cvap } 2 D}=0.6 \mu \mathrm{m}$ it represents the half of the liquid thickness in the z-axe direction.

On the figure 11 the shapes of the vaporized part and of the liquid phase are given in $r, z$ plan, on the surface the 


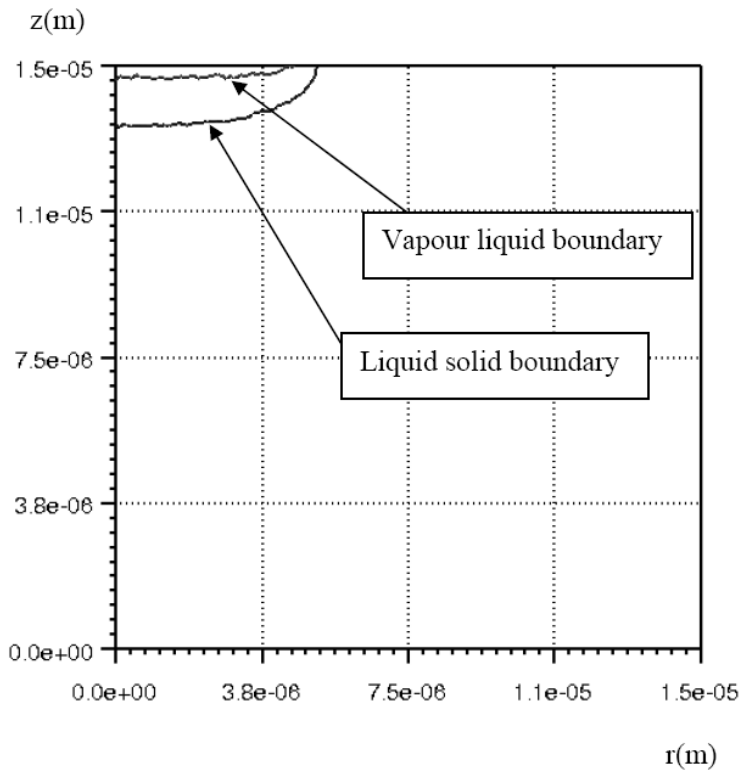

Fig. 11. A view along z-axe and r-axe of the cathode material vaporized and liquid phase at $t=100 \mathrm{~ns}$. The corresponding volumes are obtained by figure rotation along z-axe.

radius of the liquid and solid phase boundary is $r_{c l i q 2 D}=$ $5.2 \mu \mathrm{m}$. If we compare this value with the fragment radius one can see that the two values are not very different. The depth of the crater at $t=100 n s$ is $z_{\text {cvap } 2 D}=0.3 \mu m$ and the thickness of the liquid phase takes the value $z_{c l i q 2 D}-$ $z_{\text {cvap } 2 D}=1.2 \mu m$

The time evolutions of the vaporized volume and of the liquid phase are represented figure 12 the time evolution of the vaporized volume is quasi linear, with the slope value one obtain the fragment vapour erosion rate $E_{r c 2 D}=210 \mu m / C$.

\subsection{Discussion about cathode results}

Roughly the two simulations give similar results (table1) in the liquid and vapour phase apparition instants and

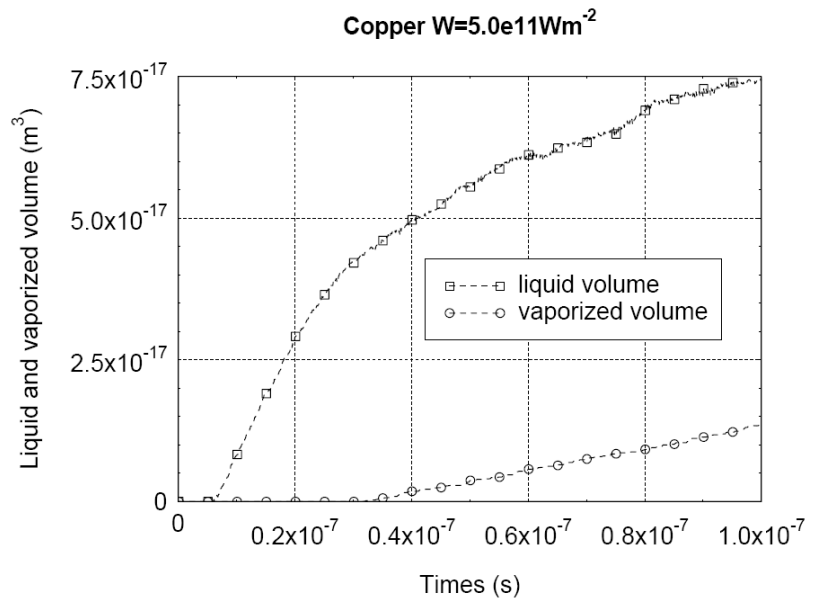

Fig. 12. Time evolution of the liquid and vaporized volume at the cathode (2D axi symmetric case).

in the evolution of the different presented curves however the discussion about the observed differences needs physical explanations. On figure 5 the vapour appears at a time $t_{\text {vap } 1 D}=25 n s$ and on figure 8 at a time $t_{\text {vap } 2 D}=32 n s$ and the energy partitions are different, this difference is the result of the energy dissipation in three possible directions in the axi symmetric case. The dissipation of the energy is different in $1 \mathrm{D}$ and $2 \mathrm{D}$ simulations, this result is also visible on figure 6 compared to figure 9. The energy dissipation is more important in $1 \mathrm{D}$ case, this fact appears distinctly in the solid phase. The figure 10 shows the difference between a cylindrical erosion (geometry defined on figure 4) and the evolution of the vaporised part and of the liquid phase; this result puts questions about the flux particle organisation [7] on the cathode surface. The curve corresponding to $20 \mathrm{~ns}$ shows clearly that in this case the metal particle flux does not exist and that the radius of the liquid part is not equal to $5 \mu m$. The paper of Jüttner [8] gives a copper erosion rate in the gap $10-300 \mu m / C$ 
Table 1. Comparison between $1 \mathrm{D}$ and $2 \mathrm{D}$ axi symmetric results at a $10 \mathrm{~A}$ cathode fragment

\begin{tabular}{lllllllll}
\hline \multicolumn{7}{c}{$t=100 n s$} \\
\hline & $t_{\text {cliq }}$ & $t_{\text {cvap }}$ & $H_{\text {vap }} / H_{\text {tot }}$ & $H_{\text {liq }} / H_{\text {tot }}$ & $H_{\text {sol }} / H_{\text {tot }}$ & $z_{\text {cliq }}-z_{\text {cvap }}$ & $z_{\text {cvap }}$ & Erosion \\
& $(n s)$ & $(n s)$ & $(\%)$ & $(\%)$ & $(\%)$ & $(\mu m)$ & $(\mu m)$ & $(\mu g / C)$ \\
\hline $1 \mathrm{D}$ & 5.4 & 25 & 50 & 29 & 21 & 1.6 & 0.4 & 415 \\
$2 \mathrm{D}$ axi & 6.1 & 32 & 20 & 26 & 54 & 1.2 & 0.3 & 210 \\
\hline
\end{tabular}

, the value $E_{r 1 D}$ is too large and the value $E_{r 2 D}$ corresponds to this interval. The measures lead to values near $40 \mu \mathrm{g} / \mathrm{C}[9]$ but the values are obtained by weighting contact electrode before and after arcing, there is a part of the vaporised material that comes back to the electrode surface or is the consequence of the anode particle flux. The cathode results show that the energy flux incoming on the cathode contact surface presents variations along the radius $\mathrm{r}$ and during the fragment live. The $1 \mathrm{D}$ simulation shows that $25 \mathrm{~ns}$ are necessary to produce vapour, this time is probably too long, the fragment existing needs the presence of metallic ions to heat the metal electrode surface. Mesyats [10] shows that a $10 A$ spot current is obtained in $0.4 n s$ with a $0.7 \mu m$ radius and a probably current density equal to $5 \times 10^{13} \mathrm{Am}^{-2}$, the $1 \mathrm{D}$ simulation shows that in this case all the incoming energy flux is used by the metal vaporisation, there is no liquid phase and no solid heating.

\section{Anode arc root}

In the electric arc the anode spot collects the column electrons, in vacuum breakers it generally appears at high cur- rent values (up to $\times 10 k A$ ) and in other breakers working at atmospheric pressure values (or up to a multiple of $0.1 M P a)$ it appears simultaneously with the first cathode fragment. In opposition to the cathode (several spots), the anode presents always one spot. JD Cobine and EE Burger [11] give a power density equation on the anode electrode surface:

$$
P_{a}=J_{a} \times\left(V_{a}+\varphi+V_{T}\right)+P_{n}+P_{r}
$$

$J_{a}$ is the anode current density, $V_{a}$ the anode drop, $\varphi$ the material work function, $P_{n}$ the result of the energy of neutral atoms and $P_{r}$ the radiant energy from the column. Usually the two last parts of equation 9 are negligible in comparison with the first part, $V_{T}$ results of electron column flux it can be taken equal to $3.0 \mathrm{~V}, \varphi$ is the material work function equal to $4.8 \mathrm{~V}$ for copper, the values taken by $V_{a}$ are generally not well defined, they can be positive or negative. The energy flux $P_{a}$ is used at the anode to increase material enthalpy, to provide emission of secondary electrons and to emit radiations like in the equation 8 used at the cathode. The same approximations can be made, so $P_{a}$ is supposed in a first time transmitted to the anode 
Copper $\mathrm{W}=5 \mathrm{e} 9 \mathrm{Wm}^{-2}$

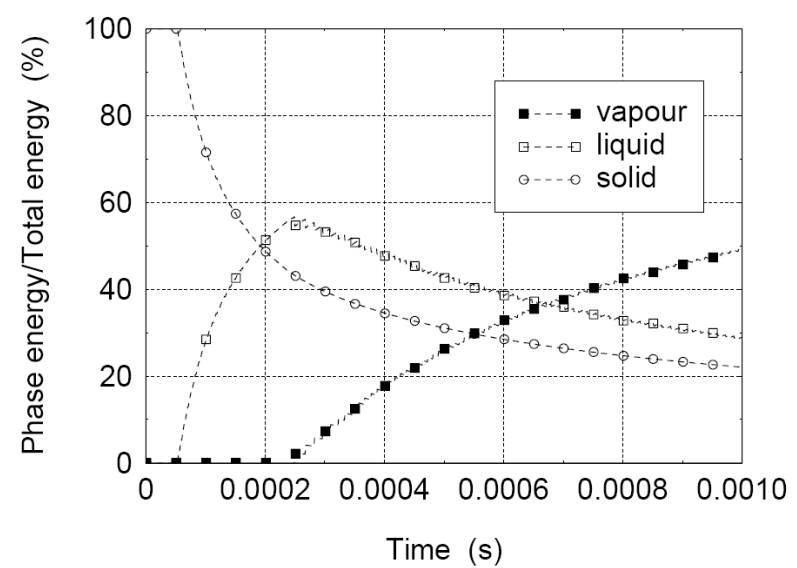

Fig. 13. The three phase energy partition versus time in the anode contact (1D case).

material. The example given here is about an anode spot carrying a $1000 A$ current. According to the values existing in $[12]$ and in [13] one find a radius anode spot $r_{a}=0.5 \mathrm{~mm}$ and a transmitted surface energy flux $W=5 \times 10^{9} \mathrm{Wm}^{-2}$. The arc duration $t_{a}=1 \mathrm{~ms}$ is a reasonable value, generally the breakers interrupt currents in some milliseconds.

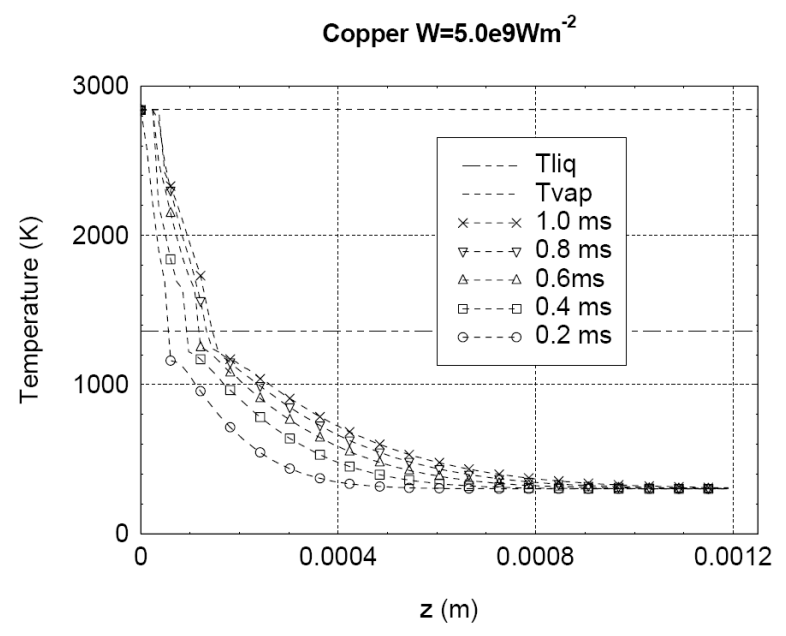

Fig. 14. Anode contact temperature repartition along $\mathrm{z}$ axe at different instants (1D case).

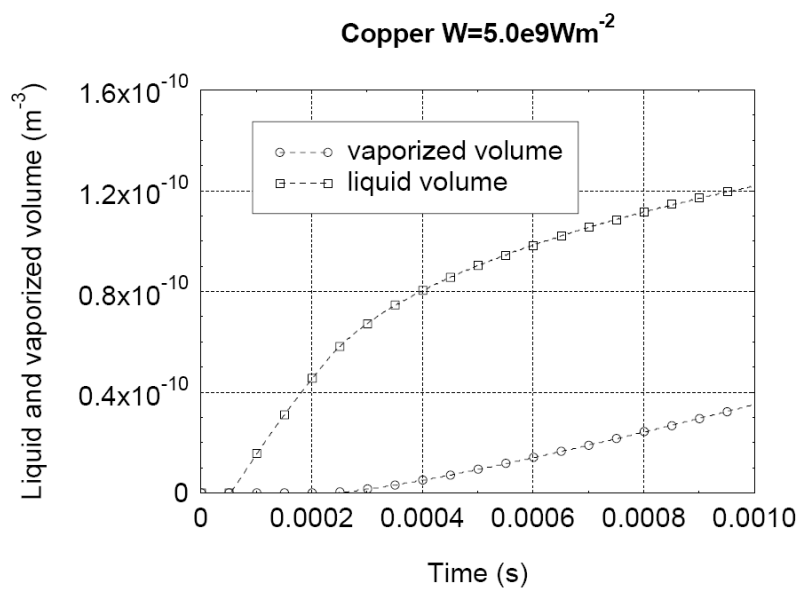

Fig. 15. Time evolution of the liquid and vaporized volume at the anode contact (1D case).

\subsection{D axi symmetric simulation results}

At $t_{\text {aliq } 2 D}=61 \mu$ s (figure 16 ) the liquid phase appears and the energy partition changes, the curve relative to liquid energy part reaches a maximum value (about 40\%) at $t_{\text {avap } 2 D}=0.34 \mathrm{~ms}$, time corresponding to the beginning of the vaporization. Then the solid phase energy part decreases slightly from $58 \%$ to $54 \%$, the liquid energy part 


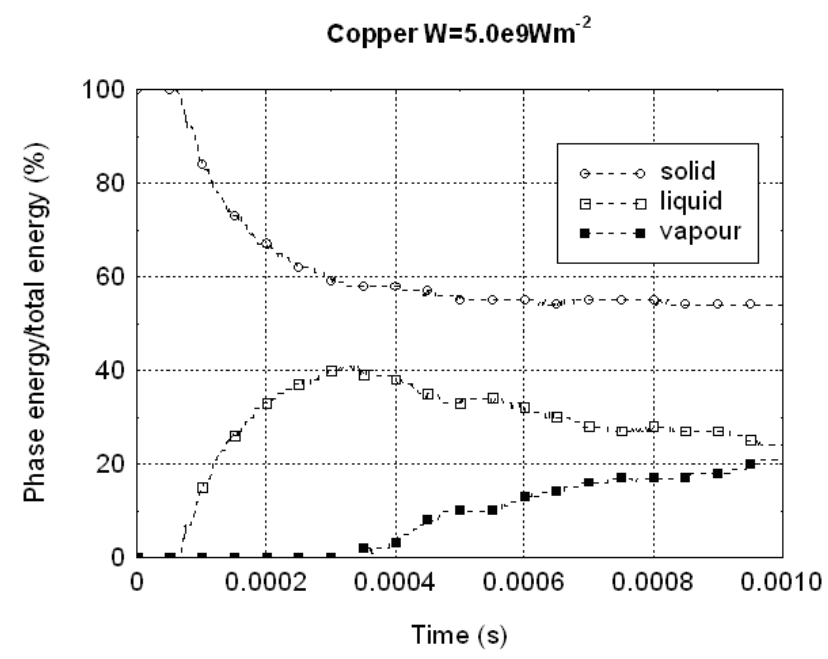

Fig. 16. The three phase energy partition versus time in the anode contact (2D axi symmetric case).

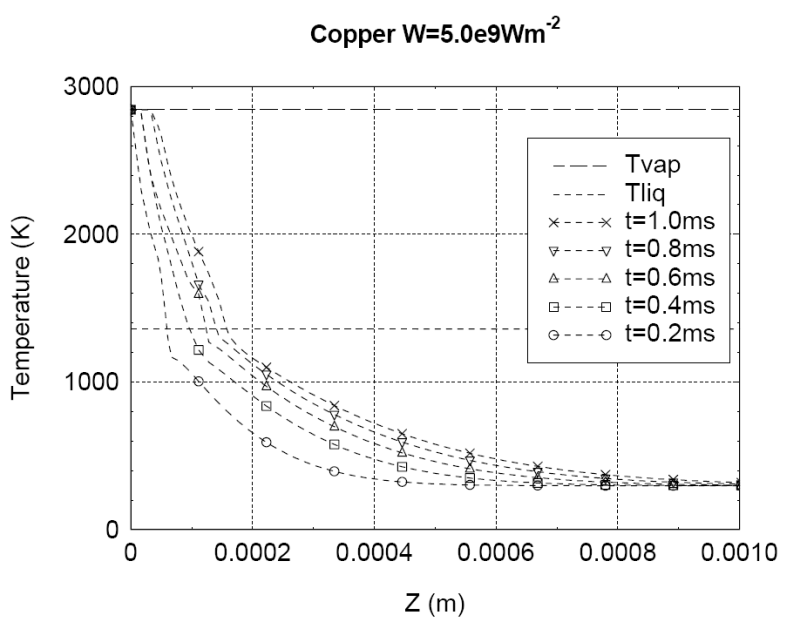

Fig. 17. Anode temperature repartition along z-axe at different instants (2D axi symmetric case).

decreases from $40 \%$ to $25 \%$ and the vapour energy part increases and is equal to $21 \%$ at $t_{a}=1 \mathrm{~ms}$.

Figure 17 presents temperature distribution along zaxe at different time values, the liquid temperature may present linear evolution generally. At $t_{a}=1 \mathrm{~ms}$ the z-axe liquid depth $z_{\text {avap } 2 D}-z_{\text {aliq2D }}=129 \mu m$ is smaller than the

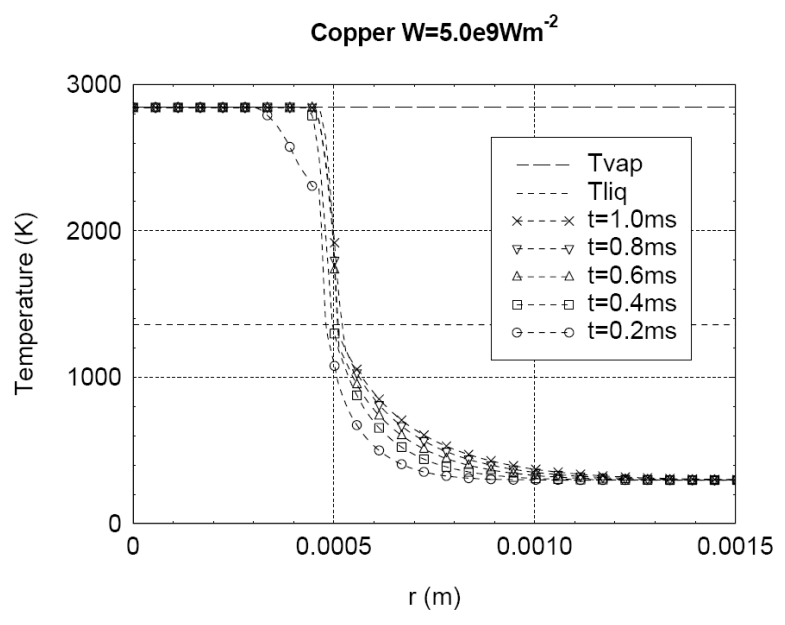

Fig. 18. Anode temperature repartition along r-axe, on contact surface at different instants (2D axi symmetric case).

$1 \mathrm{D}$ simulation depth $z_{\text {avap } 1 D}-z_{\text {aliq } 1 D}=159 \mu m$ (figure 14), the difference is $23 \%$ it is lower than cathode case.

Figure 18 shows the liquid and vapour phase establishment, at $t=0.2 \mathrm{~ms}$ the maximum radius of the liquid phase is equal to $479 \mu \mathrm{m}$.

\subsection{Discussion about anode results}

At $t_{a}=1 \mathrm{~ms}$ (figure 19), on the anode surface, the liquid and vapour phase radius are respectively equal to $520 \mu \mathrm{m}$ and $465 \mu \mathrm{m}$. The liquid phase radius is equal to the surface energy flux radius at $t=0.4 \mathrm{~ms}$. The corresponding volume evolution is given figure 20, the curve representing the vaporized volume is practically linear; this gives the erosion evaporation rate $E_{r a 2 D}=181 \mu \mathrm{g} / \mathrm{C}$.

Table 2 gives a comparison between the results obtain with the two simulation methods. The literature gives few values on the anode erosion rate, generally it is currently acknowledged that it is the same at cathode and anode; 
Table 2. Comparison between $1 \mathrm{D}$ and $2 \mathrm{D}$ axi symmetric results at a $1000 A$ anode arc root

\begin{tabular}{lllllllll}
\hline \multicolumn{1}{c}{$t=1 m s$} \\
\hline & $t_{\text {aliq }}$ & $t_{\text {avap }}$ & $H_{\text {vap }} / H_{\text {tot }}$ & $H_{\text {liq }} / H_{\text {tot }}$ & $H_{\text {sol }} / H_{\text {tot }}$ & $z_{\text {aliq }}-z_{\text {avap }}$ & $z_{\text {avap }}$ & Erosion \\
& $(\mu s)$ & $(\mu s)$ & $(\%)$ & $(\%)$ & $(\%)$ & $(\mu m)$ & $(\mu m)$ & $(\mu g / C)$ \\
\hline $1 \mathrm{D}$ & 54 & 250 & 49 & 29 & 22 & 159 & 45 & 414 \\
$2 \mathrm{D}$ axi & 61 & 340 & 21 & 25 & 54 & 129 & 31 & 181 \\
\hline
\end{tabular}

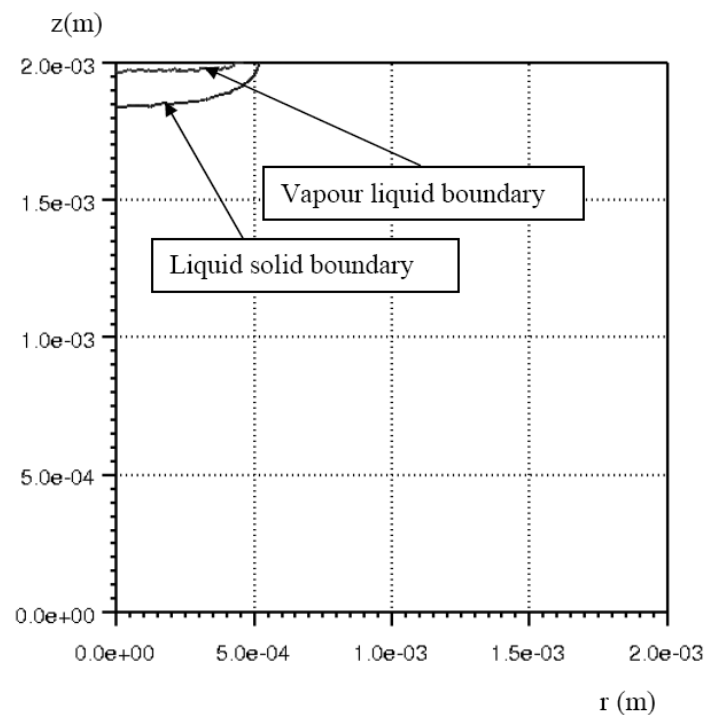

Fig. 19. A view along $\mathrm{z}$ and $\mathrm{z}$-axe of the anode material vaporized and of the liquid phase at $t=1 \mathrm{~ms}$. The corresponding volumes are obtained by figure rotation along z-axe.

however the values about silver [14] are situated in the case of a half cycle $1000 A$ current in the range $30-130 \mu \mathrm{g} / C$ and [15] find for the ratio $E_{r a} / E_{r c}$ values situated in the range $0.4-0.7$. With these parameters the anode erosion rate should be situated in the range $7-210 \mu \mathrm{g} / \mathrm{C}$ , the values given by $1 \mathrm{D}$ simulation is too large but the $2 \mathrm{D}$ simulation is in the experimental range. Like in 3.3 the obtain results gives only the erosion resulting of the vapor-

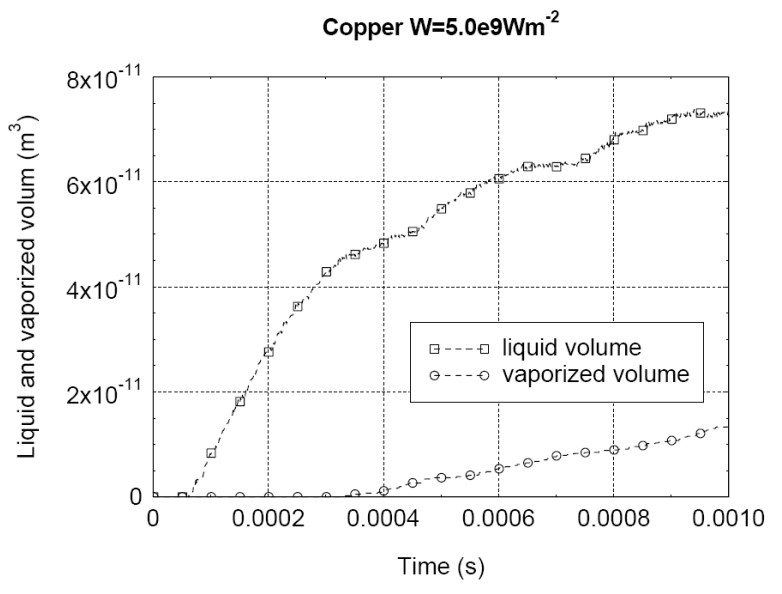

Fig. 20. Anode time evolution of the liquid and vaporized volume (2D axi symmetric case)

isation phenomena, comparison with experimental result gives only limits. The anode results are probably better than the cathode results, the reason is an evolution more simple in the anode spot live. The anode only assure the continuity of the arc current by the electron collect, the radius current density is more simple with only a transition zone on the anode edge, and its time evolution is probably monotone. 


\section{Conclusion}

Simulation of the arc effect on the copper electrodes that sustained the arc discharge have been done using two simulation possibilities the $1 \mathrm{D}$ and the $2 \mathrm{D}$ axi symmetric. The second simulation gives more precise results but the first was not without interest, it is more easy to use and it gives first results whose analysis confirms the validity of our hypothesis. The cathode fragment first nanoseconds presents a very high energy density values with only production of vapour and practically no liquid phase and no heat transfer into the solid. According to these assumptions, the 1D simulation gives a first approximation of vacuum arc ablation phenomena at small currents.

\section{References}

1. J. Rossignol., M. Abbaoui. and S. Clain, "Numerical modelling of thermal ablation phenomena due to a cathodic spot", J. Phys. D : Appl Phys. 33, (2000) pp. 2079-86

2. TH Lee and Allan Greenwood, "Theory for the Cathode Mechanism in Metal in Metal Vapour Arc", J. Appl. Phys.33 , (1961) pp. 916-23

3. TH Lee, "Energy Distribution and Cooling Effect of Electrons Emitted from an Arc Cathode", J. Appl. Phys. 31 (1960) 5 pp. 924-7

4. VI Rakhovskii "Experimental Study of the Dynamics of Cathode Spots Development", IEEE Trans. Plas. Sci. vol PS4, no 2 (1976) pp. 81-102

5. B Jüttner, "Nanosecond Displacement Times of Arc Cathode Spots in Vacuum", IEEE Trans. Plas. Sci. 27 (1999) pp. $2544-51$
6. B Jüttner and I Kleberg, "The Retrograde Motion of Arc Cathode Spots in Vacuum" J.Phys.D: Appl. Phys. 33 (2000) pp. $2025-36$

7. I Beilis "Theoretical Modelling of Cathode Spot Phenomena' Handbook of Vacuum Arc Science and Technology ed RL Boxman, PJ Martin and DM Sanders (New Jersey: Noyes) (1995) pp. 208-56

8. B Jüttner "Cathode Spots of Electric Arcs" J. Phys. D : Appl Phys. 34 (2001) R103-R123

9. JE Daalder "Components of Cathode Erosion in Vacuum Arcs" J. Phys. D : Appl Phys. 9 (1976) pp. 2379-95

10. GA Mesyats and SA Barengolts "The Cathode Spot of a High-Current Vacuum Arc as a Multiecton Phenomenon" Proc.XIXth Int. Symp. On Discharges and Electrical Insulation in Vacuum (Xian) vol 1 (2000) pp. 293-6

11. JD Cobine and EE Burger "Analysis of Electrode Phenomena in the High-Current Arc" J. Appl. Phys. 26 (1955) 7 pp. $895-8$

12. LI Sharakhovsky, A Marotta and VN Borisyuk" A Theorical and Experimental Investigation of Copper Electrode in Electrode Arc Heaters: II. The Experimental Determination of Arc Spot Parmeters" J. Phys. D : Appl Phys. 30 (1997) pp. $2018-25$

13. Ph Testé, T Leblanc and R Andlauer "A Method to Asses the Surface Power density Bought by an Electric Arc of Short Duration, and Short Electrode Gap to the Electrodes Example of Copper Electrodes" Eur. Phys. J. 18 (2002) pp. $181-8$

14. R Hemmi, Y Yokomizu and T Matsumura "Anode-fall and Cathode-fall Voltages of Air Arc in Atmosphere Between Silver Electrodes" J. Phys. D: Appl. Phys. 36 (2003) pp. 1097106 
15. J Kutzner and Z Zalucki "Electrode Erosion in the Vacuum

Arc" Proc. Int. Conf. on Gas Discharge London (1970) pp.

87-94 